\title{
EXCITABILITY CHANGES IN VENTRAL ROOT FIBERS DURING THE COURSE OF THE VENTRAL ROOT POTENTIAL
}

\author{
TOHRU GOTO* \\ Department of Physiology (1st Division), Kyoto University, Kyoto
}

The root potentials first observed by Barron and Matthews (1) are in general considered as electrotonic potentials in root fibers. Owing to their electrotonic origin, it may be expected that these potentials are accompanied by changes in the excitability in respective root fibers. In fact, Grundfest and Magnes (3) have found excitability changes in dorsal root fibers of the cat when an adjacent dorsal root was stimulated. As regards the relationship between the excitability and the potential in ventral root fibers, no experimental research has so far been reported. The present study was performed for the purpose of investigating the excitability changes which might be expected to occur in the ventral root fibers after dorsal root stimulation, thus extending our knowledge for the analysis of ventral root potential (VRP).

METHODS

All experiments were carried out on toads (Bufo vulgaris formosus). The spinal cord was severed just below the fourth ventricle and excised from the animal body with roots and a sciatic nerve of one side. In some experiments M. gastrocnemius and M. tibialis ant. were attached to the nerve. The spinal cord and roots were placed in a small airtight chamber through which moist gas consisting of $95 \% \mathrm{O}_{2}$ and $5 \% \mathrm{CO}_{2}$ was passed. The whole preparation was placed in a larger moist-chamber. The arrangement of stimulating and recording electrodes on the preparation is shown in fig. 1. The VRP's in 9th or 10th ventral root evoked by single conditioning shocks delivered to the ipsilateral dorsal root of the same segment were recorded by means of a DC or RC amplifier and a cathode-ray oscilloscope. The leading-off electrodes on the ventral root were $1 \mathrm{~mm}$ and $7 \mathrm{~mm}$ apart from the cord respectively. These electrodes were switched from the amplifier to the secondary circuit of an induction coil when test shocks were given to the ventral root fibers usually in an ascending direction.

The excitability changes were usually detected as a difference in the amplitude of diphasic action potentials in the sciatic nerve set up by test shocks of constant submaximal strength with and without conditioning dorsal root stimu-

Received for publication October 7, 1955.

* 後藤 徹 
lation. In these cases, amplitudes of action potentials were explored in advance with test shocks of varying submaximal intensities. Within a certain range of the stimulus intensity, there was found as a rule a linear relationship between both quantities (fig. 2). A stimulus intensity locating in the middle of the linearity was then chosen as a fixed intensity of the test shocks. Sometimes differences in the height of action potentials in muscle were taken as indications of the excitability change. Direct measurements of the change in threshold values were also performed in some cases. The room temperature ranged from $14^{\circ}$ to $18^{\circ} \mathrm{C}$.

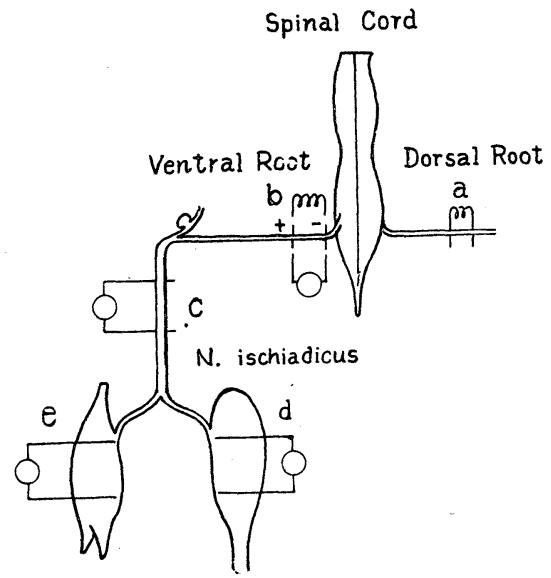

M. tibialis aut.

M. gastrocnemius

FIG. 1. Arrangement of stimulating and recording electrodes.

$a$ : Electrodes for conditioning stimulus.

$b$ : Electrodes for test-stimulus, employed also to lead VRP.

$c, d, e$ : Recording electrodes for action potentials caused by test stimulation.

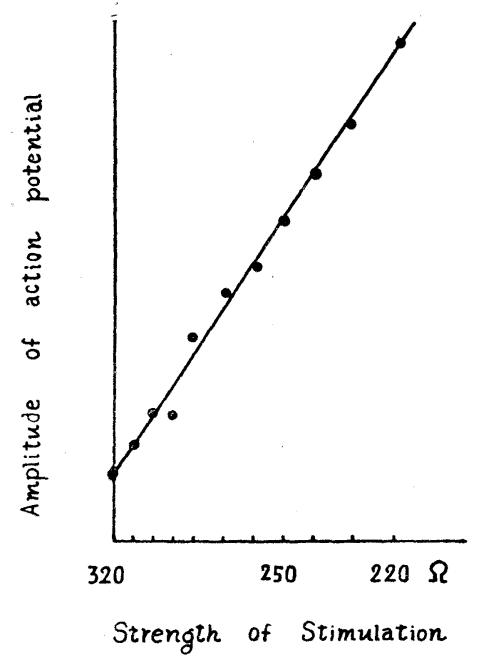

(Resistance in primary circuit of induction coil)

FIG. 2. Amplitudes of action potentials in sciatic nerve in relation to stimulus intensities given to ventral root.

\section{RESULTS}

\section{Time course of excitability change}

The increase in the excitability in ventral root fibers was readily observed during the course of VRP. An example is shown in fig. 3. In this case, the action potential in the sciatic nerve was augmented by $177 \%$ by conditioning. In the majority of cases, curves indicating the time course of the excitability change showed a sudden rise at a shock interval of 5-10 msec. and reached the maximum at an interval of less than $100 \mathrm{msec}$., until they gradually descended to the zero level as the shock interval was lengthened to 1-3 sec. (figs. 4 and $5)$. Usually, there was a small notch in the rising phase of this curve which seemed to correspond to a similar downward deflection of the potential curve. 
The whole time course of the excitability change was in good accordance with that of VRP recorded at intervals during the experiment.

Excitability curves obtained by examining action potentials in muscles (fig. 6 ) were similar to those obtained by examining sciatic nerve action potentials. The results were, however, somewhat less conclusive than the sciatic experiments probably because of the difficulty in obtaining smooth linearity in the relationship between the stimulus intensities and the size of the action potentials. Two

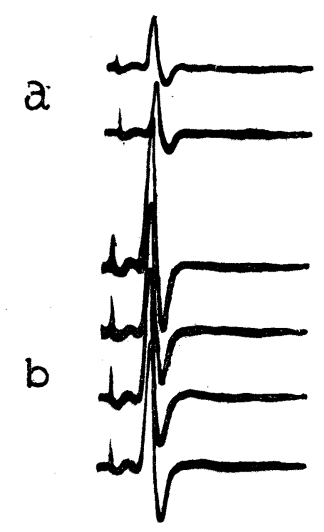
curves obtained by using antagonists, M. gastrocnemius and $M$. tibialis ant. did not always run parallel to each other (fig. 6). This fact seems to suggest some difference in the excitability and electrotonic properties between flexor and extensor neurons.

Also the direct measurement of threshold values of ventral root fibers brought about definite, but less conspicuous results (fig. 7), in accordance with what Grundfest and Magnes (3) have found on dorsal root fibers.

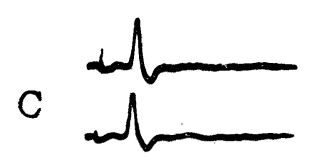

FIG. 3. Example of records showing the increased exci-

Diphasic action potentials in sciatic nerve evoked by teststimuli given to ventral root. $a$ and $c$, without conditioning. $b$, with a conditioning dorsal root stimulation preceding the test shock by $60 \mathrm{msec}$. Four records in the same condition. Augmented spike size shows the increased excitability of ventral root fibers.

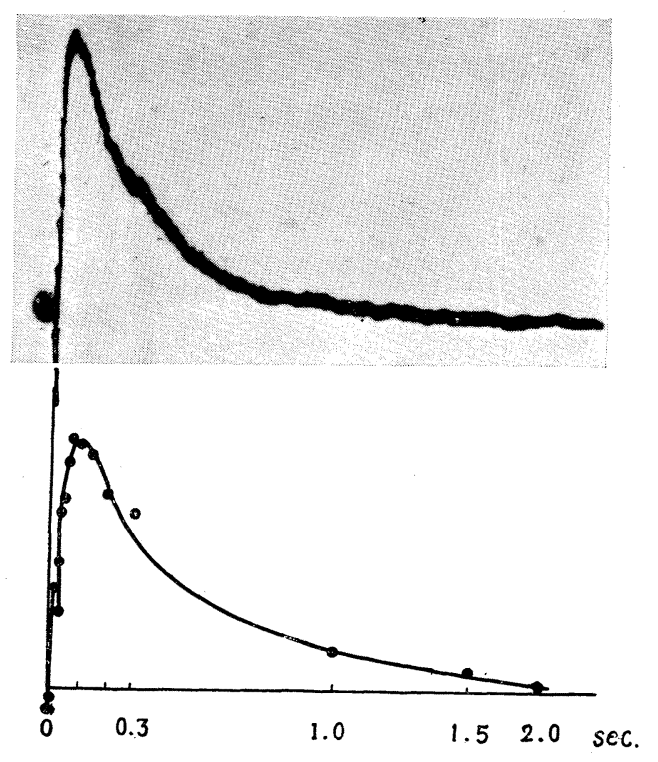

FIG. 4. Representative experiment showing the time-course of VRP and excitability change (total course). Upper curve; VRP recorded by using DC amplifier, reproduced in the same time scale as the lower curve (also in figs. 5 and 7 ). Lower curve; excitability change of tested ventral root calculated as: (height of conditioned spike)(height of unconditioned spike), the spikes being observed on sciatic nerve trunk. Increase in the excitability is shown upward. Abscissae: Intervals between conditioning and test shocks in sec. 


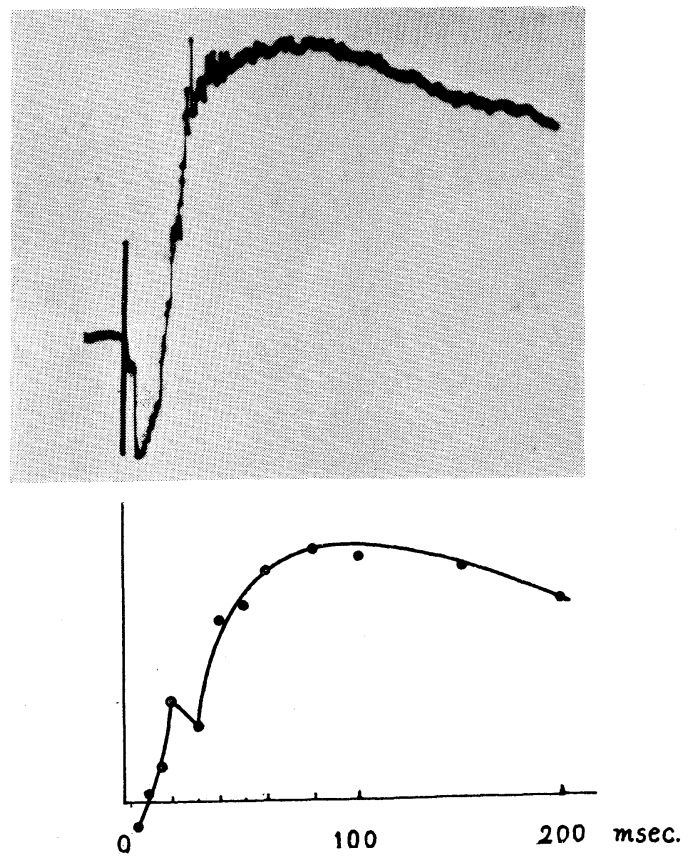

FIG. 5. Representative experiment showing the time-course of VRP and excitability changes (earlier portion of fig. 4). Abscissae: Intervals in msec. Upper curve; VRP recorded by using DC amplifier. Lower curve; excitability change. (Initial downward excursion of the upper curve was caused by leakage of cord surface potential and had little to do with electrotonic potential as well as the excitability of the ventral root.)

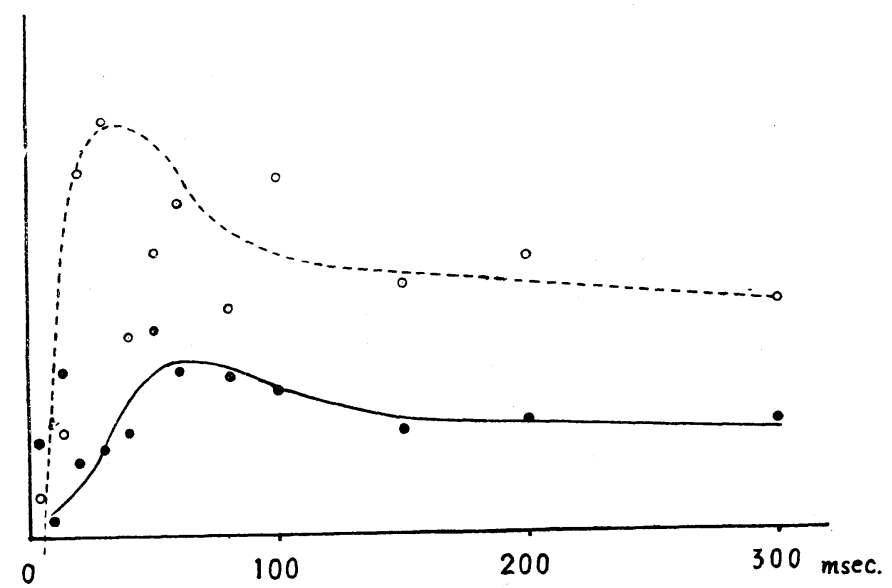

FIG. 6. The time-course of the excitability changes in ventral root measured by action potentials of two antagonistic muscles.

Open circles: M. tibialis ant. Solid circles: M. gastrocnemius. 


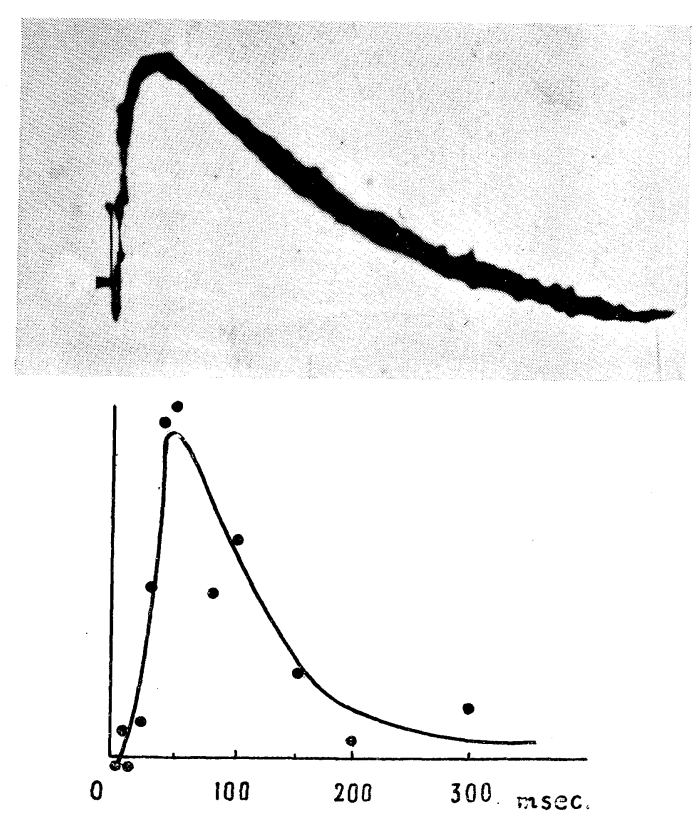

FIG. 7. The time-course of the VRP and the changes in the threshold values of ventral root fibers innervating $M$. gastrocnemius.

\section{Spatial spread of the excitability change along the ventral root}

When the distance between the cord and the point of test stimulation was increased beyond $1 \mathrm{~cm}$., any change in the excitability was no longer detected, as shown in fig. 8. If the hyperexcitability mentioned above was caused by an electrotonic potential, it might have had a spatial distribution showing an exponential decay as the distance from the cord was increased. This point was experimentally explored as follows. Test shocks, coupled with conditioning shocks

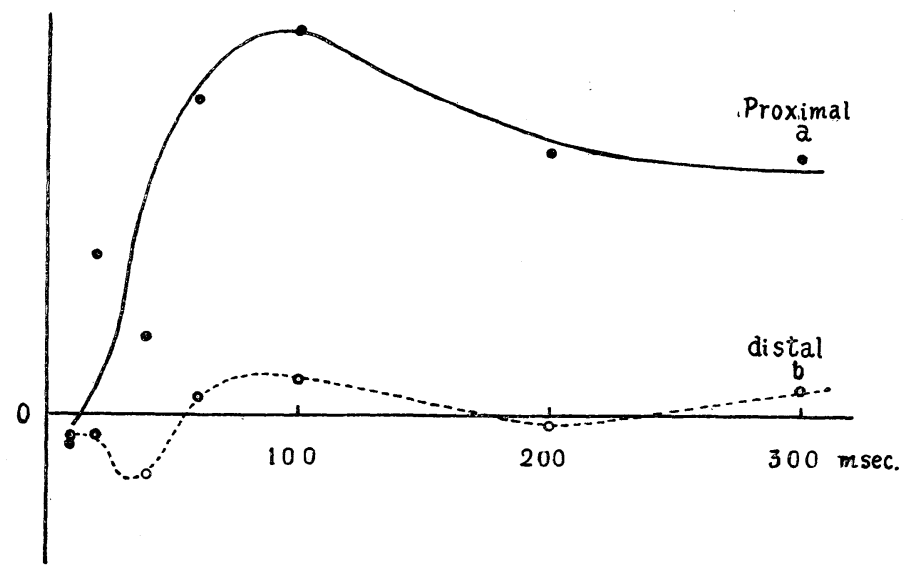

FIG. 8. Appearance of the excitability changes at a proximal point and its failure at a distal point of the ventral root.

$a$ : $1.5 \mathrm{~mm}$ from cord. $b: 11.5 \mathrm{~mm}$ from cord. 
of a certain time-interval, were given in succession to the ventral root through each pair of electrodes placed on the root at various distances from the cord. The arrangement of these electrodes is illustrated, together with the results obtained, in fig. 9. VRP's were also recorded at various distances from the cord. Both the hyperexcitability and the nega-

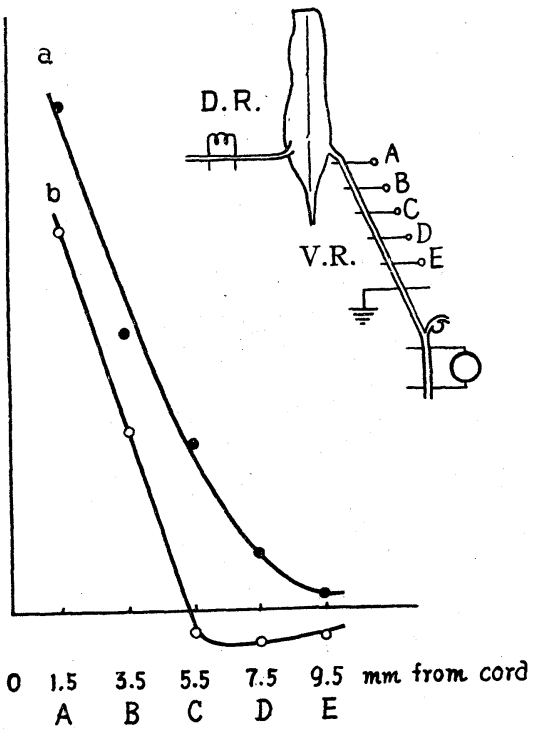
tive potential were decreased in size as the point of measurement was shifted further from the cord. As to the disparation of both curves at the distal end where a slight positive change was regularly found in the potential distribution, possible causes will be discussed below.

FIG. 9. Spatial spread along ventral root of the excitability change and VRP. Each point measured at a moment $60 \mathrm{msec}$. after dorsal root stimulation. Ordinates: Magnitudes of excitability change $(a)$ and VRP $(b)$. Abscissae: Distances from the cord to the points of observation. Inset: Arrangement of electrodes. Test stimulating cathode, as well as the proximal lead of VRP, was changed from $A$ to $E$, one by one.

\section{DISCUSSION}

The VRP is usually superimposed with spikes of reflex impulses. One may, therefore, consider that the excitability changes along the ventral root shown in the present study are, at least in part, attributable to the supernormality in the recovery cycle of ventral root fibers which have been activated reflexly by the conditioning stimulus. To exclude possible intervention of this supernormality, ample precaution was paid in providing experimental conditions suitable for minimizing reflex discharge; moderate room temperature, $\mathrm{CO}_{2}$ content of the gaseous milieu to $5 \%$, sufficient intervals of rest inserted between each stimulation, etc. Under these conditions, VRP's obtained were of so-called anelectrotonic type ( $c f$. Otani et $a l$., 5) which appeared as a slow negative deflection with less reflex discharges. Reflex discharges to this extent had in themselves only insignificant influence on the excitability of the ventral root as a whole, since no definite change was found in the excitability at a point more than $1 \mathrm{~cm}$. apart from the cord.

As shown in fig. 9, spatial distribution of the excitability change and the VRP did not run perfectly parallel to each other. This discordance seems to come chiefly from the slight positive potential at the marginal region where the negative root potential subsides. Though the mechanism of production of this positive potential is still obscure, the effect of the volume conductor ( $c f$. Bishop, 2) might be mentioned as a possible cause. Namely, at a region where only a 
small number of fibers in the ventral root conducts electrotonic currents of noticeable intensity, the ventral root may be considered as a volume conductor. According to the general rule that an electrode located near the source of current in a volume conductor leads a positive potential, the sources of the electrotonic currents in a small number of root fibers may produce a positive potential when recorded from the root surface, in reference to more distal regions. This assumption seems to have experimental support in the present research, since even in the marginal region where positive potential was recorded, the excitability of the root fibers was found always augmented and never depressed.

In view of the whole time course of the hyperexcitability keeping step with the VRP, it is reasonable to conclude that the former is caused by the catelectrotonic change set up in the root fibers by electrotonic currents. It was reported by Iwata and Otani (4) that the VRP in toads consists of two components, the slow and the spike-like. They referred the spike-like component to the post-synaptic potential in motoneuron somata, and the slow component to a long-lasting after-negativity in the terminal ramification of presynaptic neurons. The VRP's examined in the present research showed mostly predominant slow components, their electrotonic nature having been reassured. Only the initial peak in the rising phase of the excitability curve seems to correspond to the spike-like component.

\section{SUMMARY}

1. Excitability changes in ventral root fibers during the course of the VRP were examined with excised toad's spinal cord.

2. During the course of the VRP, an increase in the excitability was observed on the ventral root close to the cord. The time course of the excitability change was in accord with that of VRP recorded from the same spot.

3. Spatial spread of the hyperexcitability was examined along the ventral root. The largest increase in the excitability was found in close proximity to the cord, which declined exponentially as the test electrode was moved distally until it faded out somewhere about $10 \mathrm{~mm}$ apart from the cord. At a marginal region, distal from where any excitability change failed to appear, VRP was frequently recorded as a slight positive deflection. Even in this region an increased excitability was observed. This kind of positivity in VRP was thus accounted for by the volume conductor effect of the ventral root.

4. The results were attributed to the catelectrotonic change in the root fibers which was responsible also for the potential changes in these fibers.

The author wishes to thank Prof. T. Otani for his invaluable criticism and suggestions.

\section{REFERENCES}

1. Barron, D. H. And Matthews, R. H. C. J. Physiol. 92: 276, 1938.

2. BISHOP, G. H. Arch. Inter. Physiol. 45: 273, 1937.

3. Grundfest, H. ANd Magnes, J. Amer. J. Physiol. 164: 502, 1951.

4. Iwata, S. And Otani, T. Jap. J. Physiol. 3: 36, 1952.

5. Otani, T., Iwata, S. And Furukawa, T. Jap. J. Physiol. 2: 154, 1951 